\title{
Effect of alendronate on the mandible and long bones: an experimental study in vivo
}

\author{
Sharon R. Oyhanart ${ }^{1}$, Natalia D. Escudero ${ }^{1}$ and Patricia M. Mandalunis ${ }^{1}$
}

BACKGROUND: Bisphosphonates are anticatabolic agents that inhibit bone resorption and are widely used to treat osteoporosis and bone metastases in adults. They are also used in young patients with diseases like osteogenesis imperfecta or juvenile osteoporosis. Bone modeling/remodeling is elevated in growing subjects, and inhibition of osteoclastic activity has been shown to interfere with growth. Thus, our objective was to evaluate the effect of alendronate (ALN) on growing animals. METHODS: Healthy male Wistar rats, aged 1 mo, received ALN or vehicle for 8 wk. Serum levels (calcemia, phosphatemia, and total alkaline phosphatase) were determined. Morphometric (rat: femur and tibia weight and length and hemimandible growth) and histomorphometric parameters (thickness of tibial epiphyseal cartilage and each cartilage zone, interradicular bone volume in the first lower molar, trabeculae volume, percentage of bone and cartilage, and osteoclast number in mandibular condyles) were assessed.

RESULTS: ALN caused a significant decrease in femur and tibia length, tibial cartilage thickness, and longitudinal growth of hemimandibles. It increased interradicular bone volume and mandibular condyle trabeculae volume, increasing the percentage of cartilage and osteoclast number.

CONCLUSION: These findings indicate that administration of ALN to growing animals alters the endochondral ossification process, and thus alters growth.

B isphosphonates are synthetic analogues of pyrophosphate and are widely used in the treatment of diverse bone metabolic disorders (1-3). According to the literature, these drugs exert an anticatabolic effect by altering osteoclast recruitment, differentiation and activity, and/or by stimulating apoptosis (4-6), thus causing a decrease in bone resorption and turnover (1-3).

Based on their chemical structure, bisphosphonates can be classified as non-nitrogen-containing and nitrogen-containing bisphosphonates $(1,2)$. The latter, which include alendronate (ALN), are the most frequently used bisphosphonates at present. Once endocytosed by the osteoclast, bisphosphonates interfere with farnesyl pyrophosphate synthase, an enzyme of the mevalonate pathway, altering the posttranslational modification of key proteins in the regulation of activities that are fundamental to this cell, such as cytoskeleton organization, ruffled border formation, intracellular traffic, and survival $(3,5,7)$.

Their anticatabolic effect (1-3) and high affinity for bone $(1,2)$ make them first-choice drugs for the treatment of disorders such as osteoporosis $(1,3)$ and Paget's disease (1). They are also used in young patients $(8,9)$ to treat a number of diseases, including osteogenesis imperfecta (OI) $(1,8-11)$, juvenile osteoporosis (12), and fibrous dysplasia (13).

Bisphosphonates are used in patients with OI to treat the imbalance between bone formation and resorption and the increase in the number of osteoclasts that occur in this disease (10). Clinical trials have posited that bisphosphonates may alter growth in these patients $(11,14,15)$, and experimental studies on the subject have suggested they may be associated with a decrease in long-bone growth (16-18).

Considering that the process of endochondral ossification that takes place in long bones involves calcified cartilage matrix resorption by clastic cells for its subsequent replacement with bone, it is possible that the anticatabolic effect of a bisphosphonate may affect the ossification process, leading to a decrease in the growth of these bones. Indeed, bisphosphonates have been shown to block the removal of both bone and cartilage in growing rats, thus retarding the modeling of the metaphysis, which becomes club-shaped and radiologically denser than normal (19). This effect is also observed in children treated with high doses of bisphosphonates (20).

Unlike long bones, two different patterns of ossification occur in the mandible. Most of the body and ramus follow a direct/intramembranous ossification pattern, guided by Meckel's cartilage (21). In addition, the mandible has three pairs of secondary centers of ossification: the coronoid, symphyseal, and condylar cartilage, which ossify by indirect/endochondral ossification (21). Although the former two ossify completely before birth and during the first year of life, respectively, a remnant of condylar cartilage persists until the end of the second decade of life, providing a mechanism for growth of the mandible, in the same way as epiphyseal cartilage does in long bones $(21,22)$. The mandibular condyle has distinct features. It has a multidirectional growth capacity $(21,23)$, and although condylar growth is at its peak during puberty, condylar cartilage remains active throughout life and is able to adapt to functional demands $(23,24)$. The mandibular condyle is thus 
an important growth center of the mandible and is associated with craniofacial growth and occlusion $(22,25)$. Taking into account that it undergoes endochondral ossification (21), it can be posited that administration of a bisphosphonate would alter mandibular condyle growth.

A number of reports in the literature suggest that bisphosphonates may have an impact on the growing skeleton, causing a decrease in growth. Nevertheless, the effects of these drugs on the mandibular condyle and on long bone epiphyseal cartilage remain to be clarified. Thus, the aim of the present work was to evaluate in vivo the effect of ALN on the long bones and mandible of healthy growing rats.

\section{RESULTS}

\section{Body Weight and Body Growth}

No significant differences in body weight or growth were found either during or after treatment.

\section{Serum Parameters}

No significant differences were found in levels of calcemia, phosphatemia, or total alkaline phosphatase (Table 1).

\section{Morphometric Analysis}

Administration of ALN caused a significant decrease in the length of tibiae and femurs (Figure 1) and of OA segmenthemimandible length-in hemimandibles (Figure 2) but caused no significant change in the weight of the studied bones (Figure 1) or in any of the other segments used to assess mandibular growth (Figure 2).

Table 1. Serum determinations of calcemia, phosphatemia, and total alkaline phosphatase following treatment

\begin{tabular}{lccc}
\hline & Sham $(n=8)$ & $\operatorname{ALN}(n=7)$ & $P$ \\
\hline Calcemia $(\mathrm{mg} / \mathrm{dl})$ & $11.6 \pm 0.6$ & $11.4 \pm 1.0$ & 0.724 \\
Phosphatemia $(\mathrm{mg} / \mathrm{dl})$ & $8.6 \pm 1.2$ & $8.0 \pm 2.2$ & 0.541 \\
Total alkaline phosphatase (IU/l) & $324.4 \pm 92.6$ & $267.4 \pm 44.9$ & 0.163 \\
\hline
\end{tabular}

ALN, alendronate.
Histomorphometric Analysis

Tibial epiphyseal cartilage. Bisphosphonate treatment caused a significant decrease in the total thickness of the epiphyseal cartilage, as a result of a decrease in the thickness of both resting and proliferative cartilage zones and of hypertrophic cartilage (Figure 3).

Interradicular bone volume. The percentage of bone volume at the level of the interradicular bone of the lower first molar was significantly higher in experimental animals compared to controls (Figure 4).

Mandibular condyle. Administration of ALN caused a significant increase in mixed trabeculae volume and a higher percentage of cartilage matrix in the observed mixed trabeculae (Figure 5). Examination of hematoxylin-eosin (H\&E)stained sections confirmed a higher number of osteoclasts in the experimental group compared to controls (Figure 6) but showed no significant differences in the number of blood vessels or in total vessel volume (data not shown). It is noteworthy that the osteoclasts in the experimental group were larger and showed loss of polarization and many exhibited morphological features compatible with the apoptotic process (Figure 6).

\section{DISCUSSION}

According to the results of the present study, ALN would seemingly interfere with long-bone and mandibular growth. ALN administration resulted in a decrease in tibia and femur length and in mandibular length-segment OA according to Eratalay et al. (26). The histologic study showed a decrease in the thickness of the epiphyseal cartilage of the tibia. It also showed an increase in the volume of mixed trabeculae in the mandibular condyle, as well as in the percentage of cartilage matrix in these trabeculae; the cartilage likely persisted in the trabeculae as a result of the inhibition of osteoclast activity. The number of osteoclasts in the mandibular condyle was also found to increase.

There is limited experience in the use of bisphosphonates in children. Nevertheless, there are an increasing number of reports showing their usefulness to treat different bone

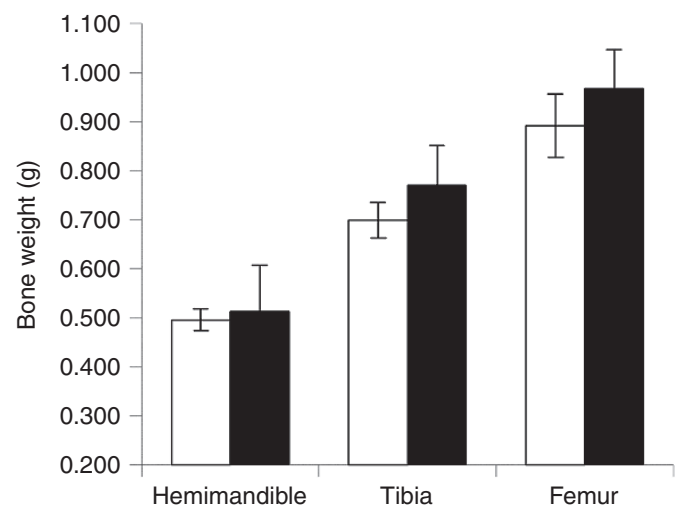

Figure 1. Bone length and weight. Sham: white columns; alendronate (ALN): black columns. Long bone length: Femur: Sham: $34.21 \pm 0.68 \mathrm{~mm}$ and ALN: $32.81 \pm 0.91 \mathrm{~mm},{ }^{*} P=0.004$; Tibia: Sham: $38.73 \pm 1.43 \mathrm{~mm}$ and $\mathrm{ALN}: 36.21 \pm 1.56 \mathrm{~mm},{ }^{*} P=0.005$. Bone weight: Hemimandible: Sham: $0.496 \pm 0.022 \mathrm{~g}$ and ALN: $0.513 \pm 0.094 \mathrm{~g}, P=0.631$; Tibia: Sham: $0.699 \pm 0.04 \mathrm{~g}$ and ALN: $0.770 \pm 0.08 \mathrm{~g}, P=0.048$; Femur: Sham: $0.891 \pm 0.065 \mathrm{~g}$ and ALN: $0.967 \pm 0.079 \mathrm{~g}$, $P=0.054$. 

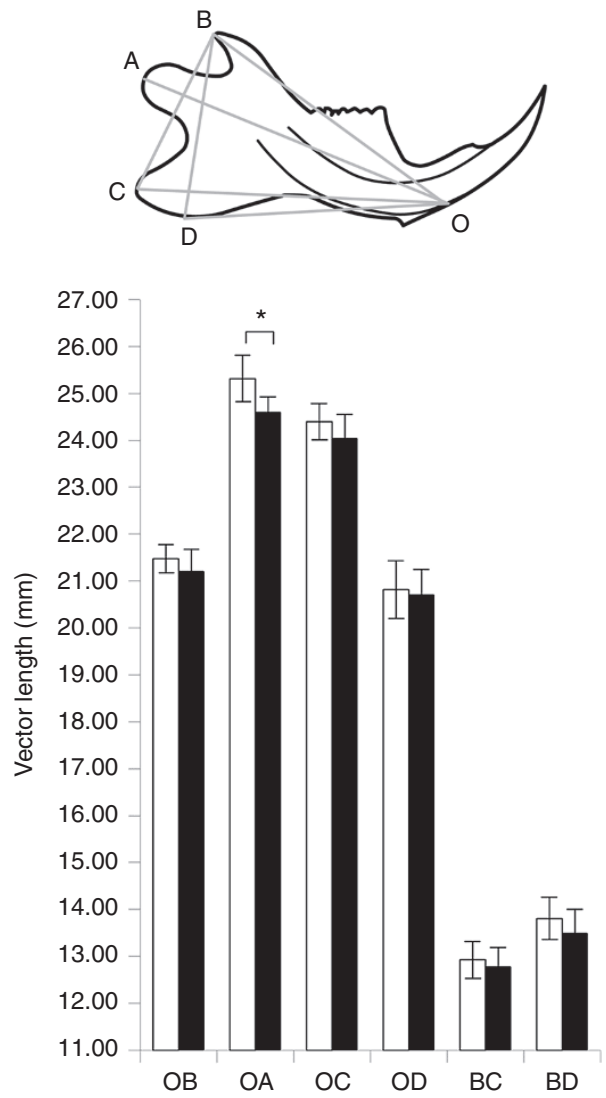

Figure 2. Vectors of mandibular growth according to Eratalay's method. Schematic drawing of a rat hemimandible showing the vectors drawn to measure mandibular growth according to Eratalay. A: middle point of the condyle. B: most superior posterior point of the coronoid process. C: most posterior point of the mandible. D: inferior border of the inferior ramus. O: most anterior inferior bone point. OA: mandibular length. OC: base. BD: height. OB: distance between points $\mathrm{O}$ and $\mathrm{B}$. OD: distance between points $O$ and $D$. BC: distance between points $B$ and C. Only segment $O A$ decreased significantly with alendronate treatment (Sham: $25.32 \pm 0.50 \mathrm{~mm}$ and ALN: $\left.24.59 \pm 0.33 \mathrm{~mm},{ }^{*} P=0.004\right)$.

metabolism pathologies that predispose children and adolescents to decreased bone mass and increased fracture risk.

These drugs have been used successfully in patients with OI. Cyclic i.v. pamidronate treatment in these patients has been shown to increase lumbar bone density $(11,15,20)$, improve vertebral geometry (11), and decrease biochemical markers of bone turnover $(11,15,20)$. At the histological level, iliac crest puncture biopsies have shown an increase in cortical width, bone volume, and number of trabeculae $(11,15)$. However, there are also reports of decreased bone formation $(11,15)$ and increased number of trabeculae with calcified cartilageprimary spongiosa $(11,15)$. Similar findings were observed in children treated with oral ALN (27-29). These clinical findings are supported by experimental animal studies on OI showing similar results, i.e., increased femoral and vertebral bone mineral density, bone volume, number of trabeculae (30), and cortical width (14).

Our findings showed that ALN caused a significant decrease in the length of long bones of healthy animals. This may partly be explained by the fact that by inhibiting bone resorption,
ALN alters the mechanism of bone modeling/remodeling at the level of the primary spongiosa, consequently affecting endochondral ossification. Our results are in contrast with reported findings in wild-type animals treated with a dose of ALN similar to the dose used in our study (30) but are partly in agreement with studies using ALN in wild-type mice (14) and clodronate in healthy rats (16). According to the results of the former study (14), only the high ALN dose $(2.5 \mathrm{mg} / \mathrm{kg}$ ) was found to affect long bone length, suggesting that low ALN doses would not result in such alterations. As to the latter study (16), involving long-term ( $32 \mathrm{wk}$ ) administration of clodronate to healthy rats, the authors found only a very high dose of the bisphosphonate $(10 \mathrm{mg} / \mathrm{kg}$ twice a week) to affect long bone length. In this regard, it must be pointed out that clodronate is 10 -fold less potent than ALN. The study presented here involved administration of a more potent drug over a shorter experimental time period, and under these experimental conditions, the effects of bisphosphonates were found to manifest sooner as compared to the aforementioned studies.

It has been shown that a decrease in chondrocyte turnover at the level of the epiphyseal cartilage leads to a decrease in bone length and an increase in cartilage area and width (31). Similar effects were reported by authors who studied the effect of ALN $(2.5 \mathrm{mg} / \mathrm{kg} / \mathrm{wk})$ on healthy growing rats (32). Our results, however, showed a marked decrease in epiphyseal cartilage thickness in ALN-treated animals, as a result of a decrease in the thickness of each of the cartilage zones (resting, proliferative, and hypertrophic). The thickness of each of the zones (hypertrophic, resting, and proliferative) relative to the total thickness was similar in treated animals (resting and proliferative zone: $68 \%$, hypertrophic zone: $32 \%$ ) and controls (resting and proliferative zone: $67 \%$, hypertrophic zone: $33 \%$ ). A possible explanation to our observations is that ALN may have a direct effect on chondrocytes via the vascular endothelial growth factor, a known regulator of angiogenesis, and essential to ossification. This growth factor also modulates chondrocyte turnover in the epiphyseal cartilage (31) and is important to chondrocyte survival (33). According to a report in the literature (34), ALN reduces levels of vascular endothelial growth factor in chondrocytes. This would explain the decrease in total cartilage thickness, particularly in the proliferative zone, as observed in the present study. Our results also showed a decrease in the thickness of the hypertrophic zone. This observation is at odds with a study (32) (2.5 mg/kg ALN dose) showing a dose-dependent decrease in hypertrophic chondrocyte apoptosis. However, our finding is partly in agreement with a report (35) that showed a decrease in the number of hypertrophic chondrocytes per column, but no changes in vascular invasion or apoptosis rate $(0.1 \mathrm{mg} / \mathrm{kg}$ ALN twice a week). It could be posited that higher doses of ALN might impair apoptosis increasing hypertrophic zone thickness, whereas lower doses would not.

The present study shows a novel finding: ALN alters mandibular growth. This alteration does not occur in all growth vectors, but specifically in the vector related with the mandibular condyle, segment OA according to Eratalay (26), one of the most important growth centers of the mandible (24). Condyle 
a

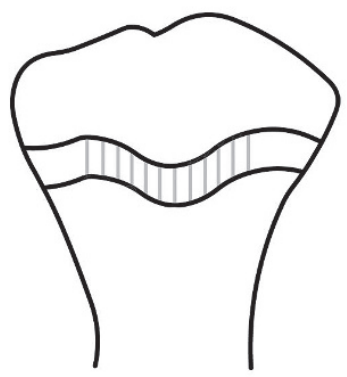

C

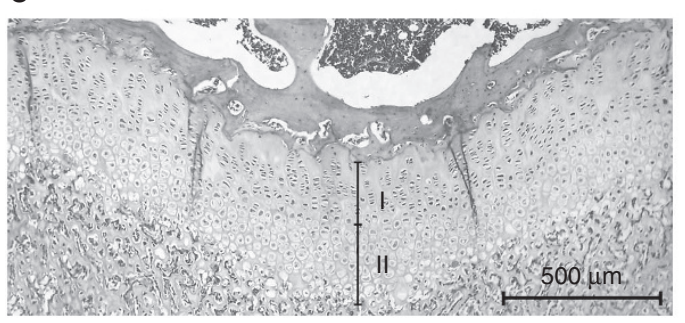

b

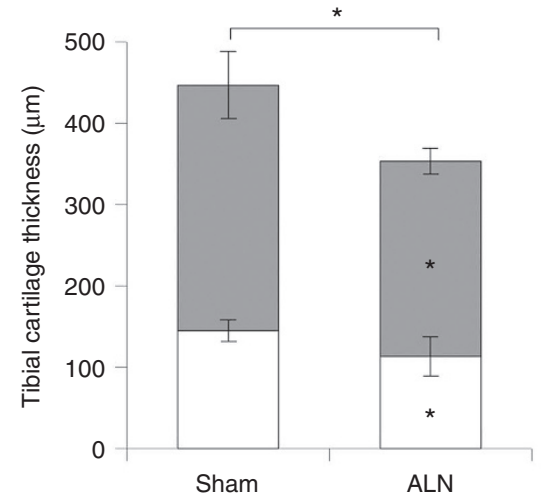

d

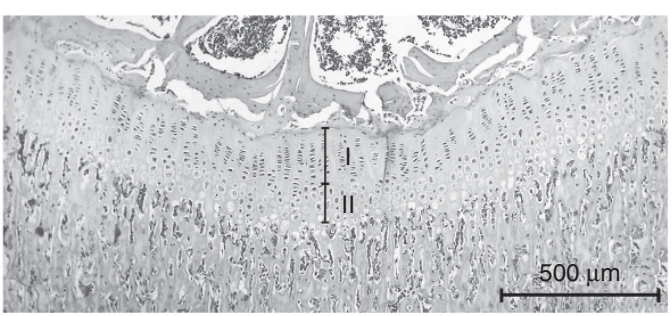

Figure 3. Changes in tibial epiphyseal cartilage. (a) Schematic drawing of a longitudinal section of the tibia showing the 12 lines used to perform epiphyseal cartilage determinations. (b) The graph shows total growth cartilage thickness and the thickness of each of the cartilage zones. Solid white corresponds to hypertrophic cartilage zone thickness (HpZ.Th, $\mu \mathrm{m})$, and the grey corresponds to resting and proliferative cartilage zone thickness (R\&PZ. Th, $\mu \mathrm{m}$ ). Gpc. Th (growth plate thickness): Sham: $447.42 \pm 49.77 \mu \mathrm{m}$ and ALN: $353.99 \pm 35.31 \mu \mathrm{m},{ }^{*} P=0.0007$. R\&PZ.Th: Sham: $302.27 \pm 41.32 \mu \mathrm{m}$ and ALN: $240.68 \pm 15.84 \mu \mathrm{m}, * P=0.004$. HpZ.Th: Sham: $145.14 \pm 13.10 \mu \mathrm{m}$ and ALN: $113.31 \pm 24.16 \mu \mathrm{m},{ }^{*} P=0.006$. (c,d) Microphotographs of H\&E-stained histologic sections showing the decrease in total epiphyseal cartilage thickness and changes in resting and proliferative zones (I) vs. hypertrophic zone (II) in an experimental animal (d) compared to a control (c). Original magnification $\times 100$. ALN, alendronate.

growth has a direct influence on the length of the ascending ramus and overall craniofacial growth, affecting intermaxillary relations and occlusion (24). There are few studies exploring the association between bisphosphonates and the mandibular condyle. It has been reported (24) that ALN treatment causes a decrease in the width of the articular cartilage and increases the size and volume of the mandibular condyle as well as the proportion of the area of the cartilage layer relative to the whole condyle (36). Our results showed that bisphosphonate treatment caused a significant increase in trabeculae volume, and a significantly higher proportion of cartilage matrix than of bone matrix in the trabeculae. This clearly reflects the anticatabolic effect of these drugs. Although a similar finding was reported previously in a descriptive study (37), it must be pointed out that the dose used by the authors was eightfold higher than the dose used here.

Though not as extensively as in long bones, the target cell of bisphosphonate therapy, the osteoclast, has also been studied in the mandibular condyle. Some authors observed a decrease in the number of osteoclasts with no differences in size compared to controls (24), whereas others found no differences in osteoclast counts in wild-type animals (36). In contrast, our study showed a significant increase in the number of clastic cells, which were also markedly larger than controls. This finding demonstrates that ALN inhibits osteoclastic activity but does not interfere in the recruitment of precursor cells, as shown by a previous study conducted at our laboratory (38). It is also worth noting that a previous study reported in the literature (32) found ALN to increase the number of osteoclasts, but to decrease cathepsin $\mathrm{K}$ staining; the latter was virtually absent in the $2.5 \mathrm{mg} / \mathrm{kg}$ dose-treated mice, showing that treatment with a nitrogen-containing bisphosphonate inhibited osteoclast maturation and resorptive capacity.

There is an interesting variability among different studies in the used dose/doses, likely due to the method used for species-to-species extrapolation of dose. Moreover, protocols for treating children are not well established although a regime of $10 \mathrm{mg} / \mathrm{d}(70 \mathrm{mg} / \mathrm{wk})$ for children weighing $>20 \mathrm{~kg}$ is accepted. A $20 \mathrm{~kg}$ body weight in humans is approximately equivalent to the weight of a prepubescent rat, which was the growth stage of the rats at the onset of the study. The dose used in our study was calculated using equations performed by other authors (39), who established that a human dose of $1 \mathrm{mg} / \mathrm{kg} /$ wk $(70 \mathrm{mg} / \mathrm{wk}$ for an average $70 \mathrm{~kg}$ human) would be equivalent to $0.35 \mathrm{mg} / \mathrm{kg} / \mathrm{wk}$ in rats.

There are limitations to our study. The experimental model used here involved only one dose of the bisphosphonate and one experimental time point. It must also be pointed out that the present study was conducted in healthy animals. Further studies using experimental models of bone disease must be conducted in order to confirm the findings reported here. In addition, using different doses and time points will contribute to a better understanding of the effects of these drugs. Moreover, further research is necessary to elucidate whether bisphosphonates have a direct effect on chondrocytes.

Beyond the limitations to our study, the findings reported here contribute to current knowledge on the effects of ALN on bone growth, particularly on mandibular growth. 
a

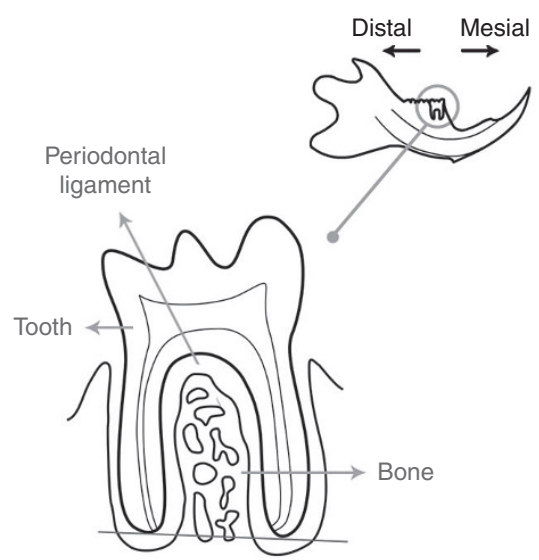

C

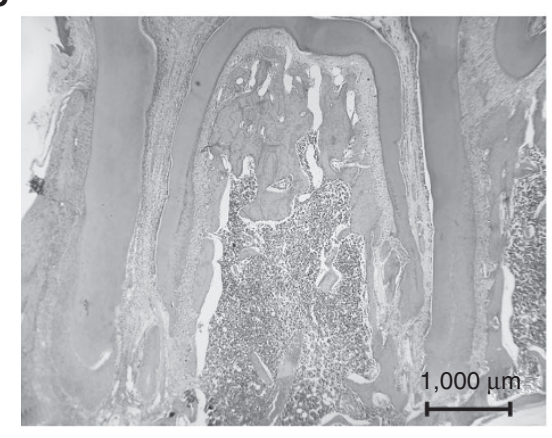

b

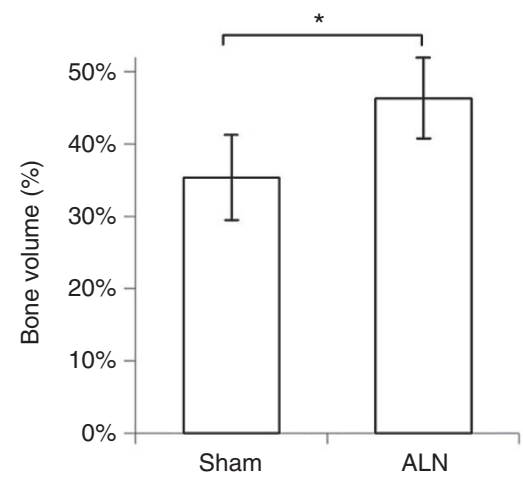

d

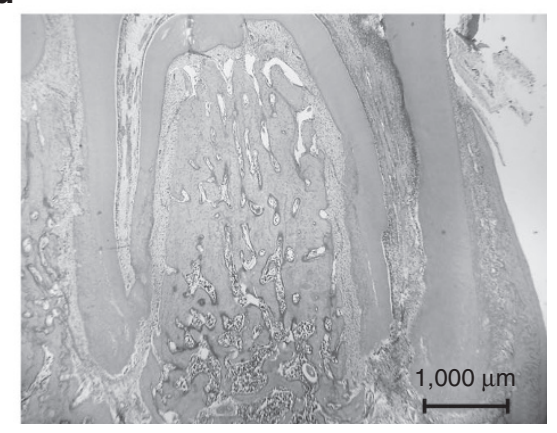

Figure 4. Interradicular bone volume. (a) Schematic drawing of rat hemimandible showing the area where the first molar is located and of a mesiodistal section of interradicular bone showing the area used to perform the histomorphometric determinations, and the neighboring structures. (b) The graph shows the percentage of bone volume present in the studied area at the level of the mandibular first molar. Sham: $35.64 \pm 5.92 \%$ and ALN: $46.32 \pm 5.60 \%$, ${ }^{*} P=0.002$. (c, d) Microphotographs of H\&E-stained histologic sagittal sections showing a greater volume of interradicular bone at the level of the lower first molar of an experimental animal (d) compared to a control (c). Original magnification $\times 40$. ALN, alendronate.

In view of the data available in the literature and the results of the present study, the beneficial effects of bisphosphonate therapy must be weighed against their negative impact on the functioning of the epiphyseal cartilage and the mandibular condyle, in cases of young patients receiving long-term treatment. The usefulness of bisphosphonates to treat pathologies like OI is well known, and these drugs have been reported to improve the quality of life of these patients. However, in other diseases such as idiopathic juvenile osteoporosis or osteoporosis secondary to other pathologies, these therapeutic agents must be used carefully. Hence, when the patient's condition allows, other currently recommended therapeutic options (12) must be considered to control the factors that may be the cause of the osteoporosis and to avoid the use of drugs that have been shown to have detrimental effects on growth.

\section{METHODS}

\section{Experimental Units and Experimental Design}

Sixteen 30 -d-old healthy male Wistar rats weighing $82.2 \pm 6.5 \mathrm{~g}$ were used. The animals were housed in groups of four per cage, under $12: 12$ light/dark cycles, lights on at $7 \mathrm{AM}$, at $18-28{ }^{\circ} \mathrm{C}$ and $52-56 \%$ humidity, and were allowed ad libitum access to food (Cooperación standard chow; Buenos Aires, Argentina) and water.

All animal procedures were performed in accordance with the 2011 8th edition of the National Research Council Guide for the Care and Use of Laboratory Animals, and with the guidelines of the ethics committee of the School of Dentistry of the University of Buenos Aires (28 November 2012-2038).

The animals were divided into two groups: Sham and experimental (ALN). Animals in the ALN group were given a weekly i.p. dose of $0.3 \mathrm{mg} / \mathrm{kg}$ of ALN (Gador S.A., Buenos Aires, Argentina), whereas Sham animals received an equivalent volume of saline solution for $8 \mathrm{wk}$. The rats were prepubescent at the beginning of the experiment and were young adults at the end of the experiment. Determination of body weight and length was performed weekly; body length was measured from tip of nose to base of tail, with the animal in prone position. At $9 \mathrm{wk}$, prior to euthanasia, the animals were anesthetized with a combination of $50 \mathrm{mg} / \mathrm{kg}$ of ketamine (Ketamine $50 \mathrm{mg} / \mathrm{ml}$ solution; Holliday-Scott S.A., Beccar, Buenos Aires, Argentina) and $0.3 \mathrm{mg} / \mathrm{kg}$ of acepromazine (Acedan; Holliday-Scott S.A.), and blood samples were obtained by intracardiac puncture for determination of calcemia, phosphatemia, and total alkaline phosphatase. After euthanasia, the tibiae, femurs, and hemimandibles were resected and fixed in buffered formalin at room temperature.

\section{Morphometric Analysis}

The tibiae, femurs, and hemimandibles were dissected and then weighed using a Mettler precision balance (E. Mettler, Zürich, Switzerland); the weight of right and left bones was averaged to obtain a single value per experimental unit (i.e., per animal).

In addition, the tibiae and femurs were measured using a vernier-type caliper, and the hemimandibles were radiographed with E speed (Eastman Kodak Company, Rochester, NY) intraoral radiographic film, using a dental X-ray unit operated at $70 \mathrm{kV}$ and $8 \mathrm{~mA}$, with a $15-\mathrm{cm}$ source to object distance and $0.8 \mathrm{~s}$ exposure time. The radiographs were digitalized using a scanner (HP Scanjet G2710). Mandibular growth of both hemimandibles was measured on the images following the method described by Eratalay et al. (26) 
a
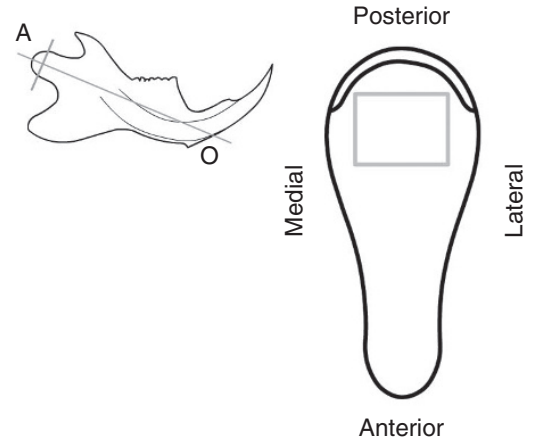

C

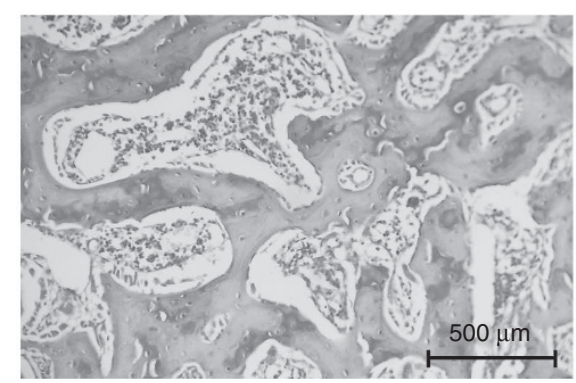

b

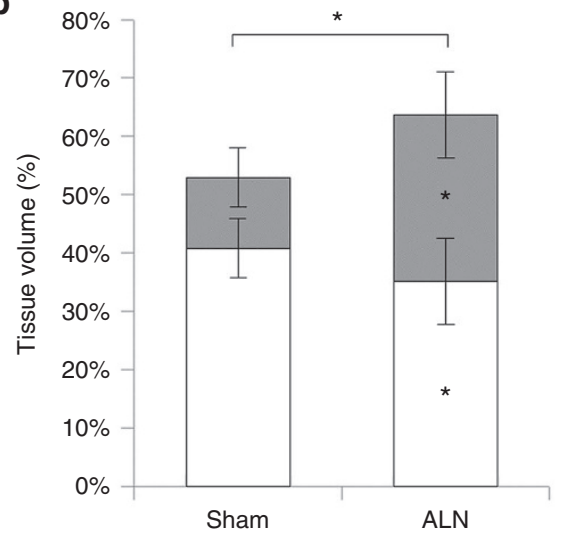

d

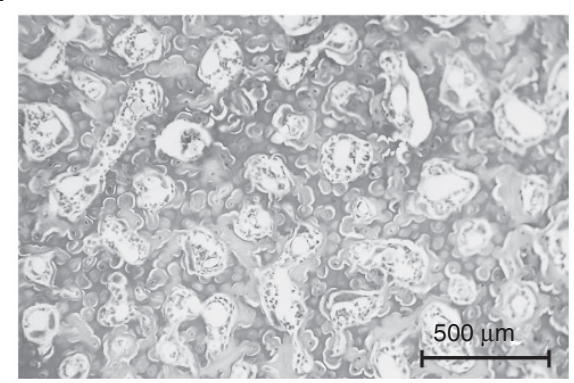

Figure 5. Changes in mandibular condyle trabecular volume. (a) Schematic drawing of a cross-section of the mandibular condyle showing the area used to measure the parameters described in the Materials and Methods section. The studied zone comprises a $0.92 \mathrm{~mm}^{2}$ area of the image; it is below the bone-cartilage interface but does not involve compact bone. (b) The graph shows the percentage of total trabeculae volume (TbV/TV, \%) in the studied area, and the percentage of bone and cartilage in the observed mixed trabeculae. The white area corresponds to bone volume (BV/TbV, \%), and the grey to cartilage volume (CgV/TbV, \%). TbV/TV: Sham: $52.94 \pm 3.40 \%$ and ALN: $63.64 \pm 0.90 \%,{ }^{*} P=0.0009$. CgV/TbV: Sham: $22.91 \pm 5.08 \%$ and ALN: $44.78 \pm 7.38 \%,{ }^{*} P=0.003$. BV/TbV: Sham: $77.09 \pm 5.08 \%$ and ALN: $55.22 \pm 7.38 \%,{ }^{*} P=0.003$. (c,d) Microphotographs of periodic acid-Schiff-stained sections of the mandibular condyle. The more intensely stained zones correspond to cartilage matrix, and the paler zones to bone matrix. A greater number of mixed trabeculae containing a higher proportion of calcified cartilage matrix, as well as a large amount of smaller medullary spaces, can be observed in an experimental animal (d) compared to a control (c). Original magnification $\times 100$. ALN, alendronate.

(Figure 2), using Image-Pro Plus 4.5.0.29 software. Results were averaged to obtain one value for each determination per animal.

\section{Histomorphometric Analysis}

Tibiae.

Following decalcification in 10\% EDTA, pH 7.4, the tibiae were processed histologically for paraffin embedding, and longitudinal sections were obtained and stained with $\mathrm{H} \& \mathrm{E}$.

\section{Hemimandibles.}

In order to study the interradicular bone at the level of the lower first molar, the hemimandibles were decalcified and sectioned mesially to the first molar and distally to the third molar. Following decalcification, mesiodistally oriented sections (Figure 4) were obtained and stained with H\&E. The condyles were dissected, and cross-sections were obtained $0.3 \mathrm{~mm}$ below the articular surface, as measured using a vernier-type caliper; the specimens were embedded in paraffin and processed histologically to obtain crosssections (Figure 5), which were stained with H\&E and periodic acid-Schiff.

Digitalized microphotographs were taken with a light microscope (Axioskop 2; Carl Zeiss, Jena, Germany) and a digital camera (Nikon CoolPix $12 \mathrm{Mp}$ ) and were analyzed using Image-Pro Plus 4.5.0.29 software to determine the following histomorphometric parameters (40):

On tibia sections:

Total epiphyseal cartilage thickness (Gpc.Th, $\mu \mathrm{m})$.

Hypertrophic cartilage thickness (HpZ.Th, $\mu \mathrm{m})$.

Resting and proliferative cartilage thickness (R\&PZ.Th, $\mu \mathrm{m}$ ).
Each of the parameters was measured along 12 straight parallel lines drawn through the cartilage $225 \mu \mathrm{m}$ apart (Figure 3 ); the 12 determinations were averaged to obtain a single value per tibia.

On interradicular bone sections:

Bone volume $(B V / T V, \%)$, defined as the proportion of total volume of the interradicular space at the level of the lower first molar (including periodontal ligament) occupied by cancellous bone.

On periodic acid-Schiff-stained mandibular condyle sections, in the area shown in Figure 5:

Trabeculae volume (TbV/TV, \%), defined as the proportion (\%) of total volume occupied by mixed trabeculae (bone + cartilage).

Percentage of bone $(B V / T b V, \%)$, defined as the proportion (\%) of total mixed trabeculae volume occupied by bone.

Percentage of cartilage ( $\mathrm{CgV} / \mathrm{TbV}, \%)$, defined as the proportion (\%) of total mixed trabeculae volume occupied by cartilage.

$\mathrm{H} \& \mathrm{E}$-stained histological sections of the mandibular condyle were studied under a light microscope (Bausch \& Lomb; Hicksville, NY) at $\times 1,000$ magnification to determine:

The number of osteoclasts per $\mathrm{mm}^{2}$ of trabecular surface (N.Oc/ $\mathrm{mm}^{2}$ ), considering all cells close to the bone surface and containing two or more nuclei.

The number of blood vessels per $\mathrm{mm}^{3}$ of bone marrow volume ( $N$. $\left.b v / \mathrm{mm}^{3}\right)$.

Total blood vessel volume ( $b v V / M V$, \%), defined as the proportion (\%) of total bone marrow volume occupied by blood vessels.

All the parameters were measured in the right and left condyles of each animal, and the results were averaged to obtain a single value for each of the eight animals (Sham: four animals; ALN: four animals). 


\section{Articles | Oyhanartet al.}

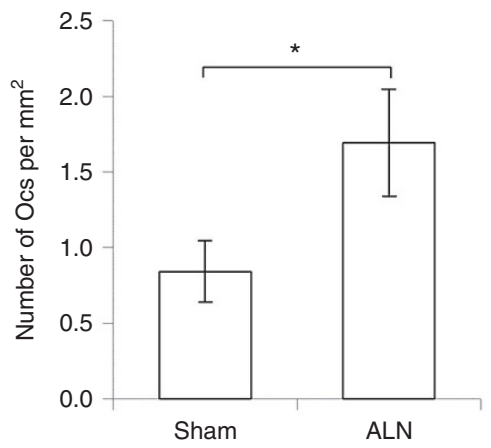

b

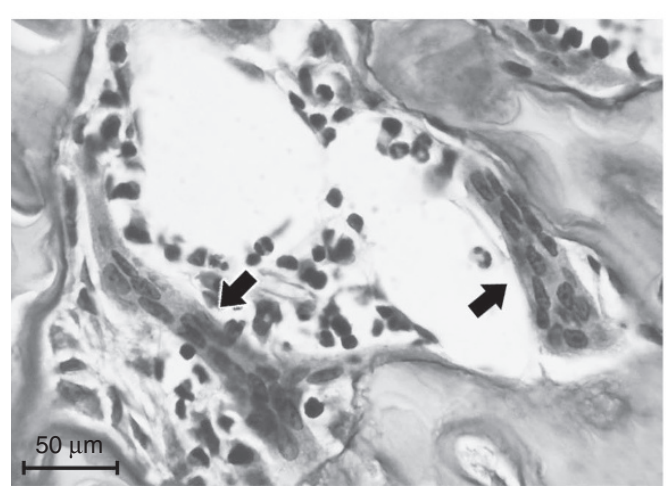

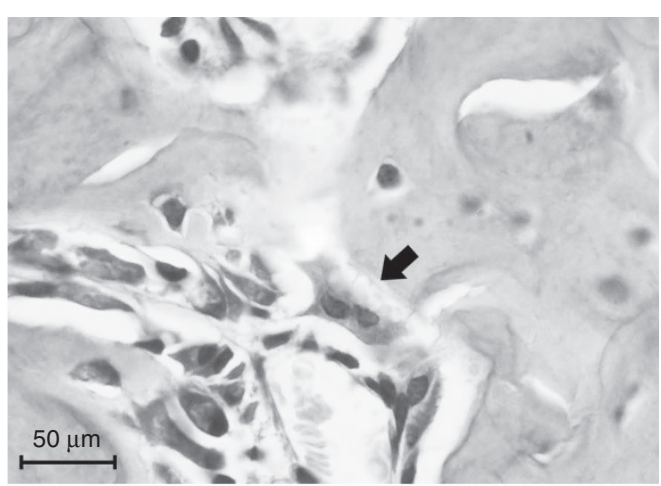

C

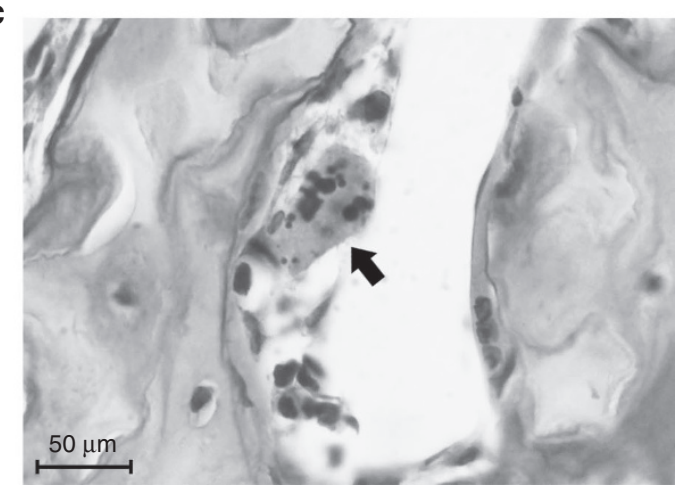

Figure 6. Osteoclasts (Oc). Alendronate caused a significant increase in the number of Oc (Sham: $0.84 \pm 0.20$ Oc $/ \mathrm{mm}^{2}$ and ALN: $1.69 \pm 0.35$ Oc/mm ${ }^{2},{ }^{*} P=$ 0.006. (a-c) Microphotographs of H\&E-stained histologic sections showing osteoclasts (black arrows) of a sham animal (a) and of experimental animals $(\mathbf{b}, \mathbf{c})$. Note the increase in Oc size and number of nuclei (b), as well as the presence of features compatible with apoptotic morphology (c) in ALN-treated animals (i.e., withdrawal from bone surface, decrease in cell size and adoption of a round shape, chromatin condensation, and initial signs of nuclei fragmentation). Original magnification $\times 1,000$. ALN, alendronate.

\section{Data Analysis}

InfoStat v2013 was employed. Shapiro-Wilks test was used to confirm normality of distribution of each of the variables in each group, and $F$-Snedecor test was applied to verify equality of variances among groups for each of the studied parameters.

All the results were expressed as mean and SD and were statistically analyzed using Student's paired t-test, and Satterthwaite's correction was applied when the analyzed variable had normal distribution but variances were unequal.

\section{ACKNOWLEDGMENTS}

The authors thank GADOR S.A. for supplying the alendronate, Vet Marianela Lewicki for her expert assistance in animal care and in handling animals during procedures, Laboratory Technicians Mariela Lacave and Ivana Sánchez Rojas for processing the samples and obtaining the histological sections, and Lic. Ariel Gualtieri for his assistance in performing the statistical analyses.

\section{STATEMENT OF FINANCIAL SUPPORT}

This study was supported by UBACyT 20020120100190BA.

Disclosures: The authors declare that there are no conflicts of interest.

\section{REFERENCES}

1. Drake MT, Clarke BL, Khosla S. Bisphosphonates: mechanism of action and role in clinical practice. Mayo Clin Proc 2008;83:1032-45.

2. Russell RG. Bisphosphonates: the first 40 years. Bone 2011;49:2-19.

3. Kavanagh KL, Guo K, Dunford JE, et al. The molecular mechanism of nitrogen-containing bisphosphonates as antiosteoporosis drugs. Proc Natl Acad Sci USA 2006;103:7829-34.

4. Benford HL, McGowan NW, Helfrich MH, Nuttall ME, Rogers MJ. Visualization of bisphosphonate-induced caspase- 3 activity in apoptotic osteoclasts in vitro. Bone 2001;28:465-73.
5. Van Beek ER, Löwik CW, Papapoulos SE. Bisphosphonates suppress bone resorption by a direct effect on early osteoclast precursors without affecting the osteoclastogenic capacity of osteogenic cells: the role of protein geranylgeranylation in the action of nitrogen-containing bisphosphonates on osteoclast precursors. Bone 2002;30:64-70.

6. Sudhoff H, Jung JY, Ebmeyer J, Faddis BT, Hildmann H, Chole RA. Zoledronic acid inhibits osteoclastogenesis in vitro and in a mouse model of inflammatory osteolysis. Ann Otol Rhinol Laryngol 2003;112(9 Pt 1):780-6.

7. Luckman SP, Hughes DE, Coxon FP, Graham R, Russell G, Rogers MJ. Nitrogen-containing bisphosphonates inhibit the mevalonate pathway and prevent post-translational prenylation of GTP-binding proteins, including Ras. J Bone Miner Res 1998;13:581-9.

8. Sebestyen JF, Srivastava T, Alon US. Bisphosphonates use in children. Clin Pediatr (Phila) 2012;51:1011-24.

9. Srivastava T, Alon US. The role of bisphosphonates in diseases of childhood. Eur J Pediatr 2003;162:735-51.

10. Forlino A, Cabral WA, Barnes AM, Marini JC. New perspectives on osteogenesis imperfecta. Nat Rev Endocrinol 2011;7:540-57.

11. Rauch F, Travers R, Glorieux FH. Pamidronate in children with osteogenesis imperfecta: histomorphometric effects of long-term therapy. J Clin Endocrinol Metab 2006;91:511-6.

12. Bachrach LK, Ward LM. Clinical review 1: bisphosphonate use in childhood osteoporosis. J Clin Endocrinol Metab 2009;94:400-9.

13. Lee JS, FitzGibbon EJ, Chen YR, et al. Clinical guidelines for the management of craniofacial fibrous dysplasia. Orphanet J Rare Dis 2012;7:Suppl $1:$ S2.

14. Evans KD, Lau ST, Oberbauer AM, Martin RB. Alendronate affects long bone length and growth plate morphology in the oim mouse model for Osteogenesis Imperfecta. Bone 2003;32:268-74. 
15. Munns CF, Rauch F, Travers R, Glorieux FH. Effects of intravenous pamidronate treatment in infants with osteogenesis imperfecta: clinical and histomorphometric outcome. J Bone Miner Res 2005;20:1235-43.

16. Koivukangas A, Tuukkanen J, Hannuniemi R, Jämsa T, Kippo K, Jalovaara P. Effects of long-term administration of clodronate on growing rat bone. Calcif Tissue Int 2001;69:350-5.

17. Lepola VT, Hannuniemi R, Kippo K, Lauren L, Jalovaara P, Vaananen HK. Long-term effects of clodronate on growing rat bone. Bone 1996;18: 191-6.

18. Pataki A, Müller K, Green JR, Ma YF, Li QN, Jee WS. Effects of shortterm treatment with the bisphosphonates zoledronate and pamidronate on rat bone: a comparative histomorphometric study on the cancellous bone formed before, during, and after treatment. Anat Rec 1997;249:458-68.

19. Schenk R, Merz WA, Mühlbauer R, Russell RG, Fleisch H. Effect of ethane-1-hydroxy-1,1-diphosphonate (EHDP) and dichloromethylene diphosphonate ( $\mathrm{Cl} 2 \mathrm{MDP}$ ) on the calcification and resorption of cartilage and bone in the tibial epiphysis and metaphysis of rats. Calcif Tissue Res 1973;11:196-214.

20. Glorieux FH, Bishop NJ, Plotkin H, Chabot G, Lanoue G, Travers R. Cyclic administration of pamidronate in children with severe osteogenesis imperfecta. N Engl J Med 1998;339:947-52.

21. Nanci A. Ten Cate's Oral Histology: Development, Structure, and Function. 8th edn. St Louis, MO: Mosby Elsevier, 2008:32-56.

22. Profitt WR, Fields HW, Sarver DM. Contemporary Orthodontics. 4th edn. St Louis, MO: Mosby Elsevier, 2006.

23. Owtad P, Park JH, Shen G, Potres Z, Darendeliler MA. The biology of TM] growth modification: a review. J Dent Res 2013;92:315-21.

24. Kim MS, Jung SY, Kang JH, et al. Effects of bisphosphonate on the endochondral bone formation of the mandibular condyle. Anat Histol Embryol 2009;38:321-6.

25. Enlow DH, Moyers RE. Handbook of Facial Growth. 2nd edn. Philadelphia, PA: Saunders, 1982.

26. Eratalay YK, Simmons DJ, El-Mofty SK, et al. Bone growth in the rat mandible following every-day or alternate-day methylprednisolone treatment schedules. Arch Oral Biol 1981;26:769-77.

27. Cho TJ, Choi IH, Chung CY, Yoo WJ, Park MS, Park YK. Efficacy of oral alendronate in children with osteogenesis imperfecta. J Pediatr Orthop 2005;25:607-12.

28. Vyskocil V, Pikner R, Kutílek S. Effect of alendronate therapy in children with osteogenesis imperfecta. Joint Bone Spine 2005;72:416-23.
29. Seikaly MG, Kopanati S, Salhab N, et al. Impact of alendronate on quality of life in children with osteogenesis imperfecta. J Pediatr Orthop 2005;25:786-91.

30. Uveges TE, Kozloff KM, Ty JM, et al. Alendronate treatment of the brtl osteogenesis imperfecta mouse improves femoral geometry and load response before fracture but decreases predicted material properties and has detrimental effects on osteoblasts and bone formation. J Bone Miner Res 2009;24:849-59.

31. Gerber HP, Vu TH, Ryan AM, Kowalski J, Werb Z, Ferrara N. VEGF couples hypertrophic cartilage remodeling, ossification and angiogenesis during endochondral bone formation. Nat Med 1999;5:623-8.

32. Evans KD, Sheppard LE, Grossman DI, Rao SH, Martin RB, Oberbauer AM. Long Term cyclic pamidronate reduces bone growth by inhibiting osteoclast mediated cartilage-to-bone turnover in the mouse. Open Orthop J 2008;2:121-5.

33. Zelzer E, Mamluk R, Ferrara N, Johnson RS, Schipani E, Olsen BR. VEGFA is necessary for chondrocyte survival during bone development. Development 2004;131:2161-71.

34. Evans KD, Oberbauer AM. Alendronate inhibits VEGF expression in growth plate chondrocytes by acting on the mevalonate pathway. Open Orthop J 2009;3:83-8.

35. Zhu ED, Louis L, Brooks DJ, Bouxsein ML, Demay MB. Effect of bisphosphonates on the rapidly growing male murine skeleton. Endocrinology 2014;155:1188-96.

36. Kimura M, Miyazawa K, Tabuchi M, Maeda H, Kameyama Y, Goto S. Bisphosphonate treatment increases the size of the mandibular condyle and normalizes growth of the mandibular ramus in osteoprotegerin-deficient mice. Calcif Tissue Int 2008;82:137-47.

37. Bradaschia-Correa V, Barrence FA, Ferreira LB, Massa LF, AranaChavez VE. Effect of alendronate on endochondral ossification in mandibular condyles of growing rats. Eur J Histochem 2012;56:e24.

38. Escudero ND, Mandalunis PM. Influence of bisphosphonate treatment on medullary macrophages and osteoclasts: an experimental study. Bone Marrow Res 2012;2012:526236.

39. Huang RC, Khan SN, Sandhu HS, et al. Alendronate inhibits spine fusion in a rat model. Spine (Phila Pa 1976) 2005;30:2516-22.

40. Dempster DW, Compston JE, Drezner MK, et al. Standardized nomenclature, symbols, and units for bone histomorphometry: a 2012 update of the report of the ASBMR Histomorphometry Nomenclature Committee. J Bone Miner Res 2013;28:2-17. 